unit characters represented probably by some tangible protoplasmic unit which is transmissible from cell to cell and in sexual unions has become a dominant idea. It is very important that careful cytological studies be made of hybrids and their parents and of mutations, to determine so far as possible the protoplasmic mechanism of heredity. It will be the aim of the Committee on Heredity to encourage work on the various important problems and from time to time present papers summarizing the knowledge on the various important factors.

Appended to the present report are short statements regarding some of the important problems by Dr. C. B. Davenport and Professor W. J. Spillman, which are submitted for publication following this report. Respectfully submitted,

C. W. WARD, Chairman of Committee.

\title{
RECENT ADVANCES IN THE THEORY OF BREEDING.
}

By Dr. Charles B. Davenport, Cold Spring Harbor, New York.

The new era in theoretical breeding opened in 1900 with the rediscovery of Mendel's law. At the same time something even more important was disclosed; namely, that Devries, Porrens, Tschermak, Spillman and still others were already advanced in extensive, accurate, and even statistical studies of inheritance in plants and animals. The rediscovery of Mendel's law was no accident; it was the necessary consequence of the new studies, and similarly these new investigations were the natural growth of a time fully ripe for them.

The apparent difficulties of pedigree breeding had deterred many scholars who realized its importance from attempting it, but after the task was actually undertaken its difficulties were seen to have been overestimated. At the present time even in universities, where there is no school of agriculture, excellent work in breeding is being done, for instance by Devries at Amsterdam; by Bateson and his pupils at the University of Cambridge, England; by Correns at the University of Leipsic; by Cuenot at the University of Nancy; and in America by Castle at Harvard, by Morgan and Crampton at Columbia, by Whitman and Tower at Chicago, by Moenkhaus at Indiana, and by Kellogg and Miss MacCracken at Stanford. Pedigree breeding at the nonagricultural universities has developed greatly in the last five years, a fact that promises well for the future.

The agricultural schools are at last awakening to their opportunity to contribute to research on the laws of heredity. Abroad such work is being done by Tschermak at the Agricultural College of Vienna, by Plate at Berlin, by Toyama at Tokyo; and in this country by Gowell at Maine, Rice at Cornell, and Erf at Kansas, all working with poultry, and by scores of others working with various groups of animals and plants. Nor must we overlook the numerous independent seientific breeders, like Alexander Graham Bell on his Nova Scotia sheep farm. 
At the same time societies of broad scope have been established. Thus, in addition to the American Breeders' Association and the International Congress of Plant Hybridization, there has recently been established the Deutches Gesellschaft für Züchtungs kunde. Thus the number of workers and their organizations are fairly satisfactory. The scientific results of experimental investigations in breeding are now multiplying rapidly. Mendel's law is coming everywhere to be recognized as the starting point for studies upon inheritance. The newer investigations are, however, revealing certain important accessory and modifying laws which deserve a more detailed consideration.

First.-Mendel's law of dominance is not universal; it holds primarily for non-blending of alternative characters. Numerous allomorphic characteristics form a mosaic or even blend in the offspring. Thus, Castle has shown that the length of the ear in the young of shorteared crossed with lop-eared rabbits is intermediate between the parents and of the intergrading there may be all degrees. Similarly in poultry the height of the nostril and the degree of booting on the feet thus blend.

Second.-Reversion is a phenomenon that has long been noticed and which was studied by Darwin. He spoke of reversion as though it was in opposition to the usual laws of inheritance, which in hybridization are transcended as it were by a new law of return to an ancestral condition. It is, however, now becoming clear that reversion is no infraction of the ordinary laws of inheritance but constitutes a special case of inheritance in hybridization. In the color of animals it usually results from the crossing of a black with a light, revealing red or a hidden pattern. Either the pattern is hidden in the black by an excess of melanic pigment, or else it is latent in the white parent, requiring some pigment to bring it into view. For example, when a black spanish cock is crossed with an albino-plumaged silky, the offspring are black and red, the red not being visible in either parent. Yet, the areas occupied by the red can be distinguished in the black plumage by peculiar properties of the black in these areas. The result is as though the deficiency of a pigment in the albinic silky canceled the excess of black in the Spanish plumage; revealing the hidden red. Many other cases of reversion may be similarly explained.

Third.-The Mendelian law takes no account of the immediate characters of the parents, but only of their ancestry. Hybrids $a$ and $b$ may be very unlike in given characteristics, but if they have the same ancestry, they should (according to the Mendelian formula), when mated with a hybrid male of the same origin as themselves, each produce offspring in which the various grandparental characteristics recur in the same proportions. My own studies, still unpublished, do not confirm this dogma. On the contrary, from the somatic condition of the hybrid mother, much may be predicted as to the parentage distribution of the characters in the offspring. For example, when a singlecombed bird is crossed with a V-combed bird, the hybrids all have 
$\mathrm{Y}$-combs, but the proportional length of the arms of the $\mathrm{Y}$ to the stem varies from 5 to 95 per cent. If two females, one with a 5 per cent split and the other with a 95 per cent split be mated to a singlecomb male, Mendelian expectation is 50 per cent split comb and 50 per cent single in the offspring of either female. But actually the female with the slightly split comb will throw an excess of single-combed while the female with a deeply split comb will throw a deficiency of single-combed offspring. The average of the progeny of $a$ and $b$ together may well be 50 per cent single, but the percentage in the offspring of each form of comb will differ greatly in the two cases and the percentage can be approximately inferred from the somatic condition of the immediate parents.

Indeed it has become daily more apparent that from an observation of the soma much can be inferred as to the contents of the germ glands. As is well known in hybridization experiments, all of the offspring of the first hybrid generation usually inherit only one (the dominant) form of two allomorphs. In the second hybrid generation, we get 25 per cent with the recessive form, which henceforth breeds true and 75 per cent with the dominant. Of this 75 per cent, one-third are pure dominants and when bred inter se breed true and two-thirds are heterozygous, forming gametes of both kinds. It is usually. stated that the pure dominants cannot be distinguished from the heterozygous dominants except by breeding tests, but this is usually not true, for one who is well acquainted with his material will usually observe differences in the appearance of the two kinds such that he can quickly select the pure dominants. Thus when a white leghorn is crossed with a Black Minorca the offspring are all white with scattered black feathers. If these offspring be mated together 25 per cent black offspring appear and 75 per cent white. But of the $\mathbf{7 5}$ per cent white, one-third will be pure white and the rest will have black specks. The pure white will usually be found to be homozygous. Thus the heterozygous form has the hybrid nature of its germ cells stamped upon its soma.

Now that we recognize that many of the characters of plants and animals are of the order of mutations, it remains to investigate methods of inducing mutations as fluctuations have long been induced. Our colleague, Dr. MacDougal, has succeeded in this line of work by injecting into the ovaries of plants certain salts. In how far this or other methods of inducing mutations may be extended remains to be seen, but it is clear that the experiment deserves to be tried immediately on other materials.

More and more it becomes clear that problems of inheritance are chemical problems, and that the development of this or that characteristic of the adult is due to the presence of a specific enzyme in the germ cells. Thus an albino mouse may be derived from a gray mouse, a black mouse, or a chocolate mouse. Now albinos of whatever origin when bred inter se produce always albinos. But if an albino that has been extracted from a gray be bred to a colored mouse (e. g. chocolate), 
the gray will appear in all the offspring, or if the albino were one extracted from a black, the offspring will all be black. The albinos look alike only because they lack one or two color-producing factors. When the lacking factor is supplied by a pigmental animal of any sort, the particular ancestral color appears again. Similarly in poultry the germ cells of an albino plumage, such as the silky has, behave very differently in inheritance from the dominant white as seen in the white leghorn, and this difference in behavior of the two whites corresponds to a difference in their chemical composition. Indeed the fact, that the enzymes of the germ cells and particularly of the egg determine hereditary characters, points the way to the modification of hereditary qualities, and to the production of this or that character at will. Such, at least, is the goal of the investigator.

\section{THE CHROMOSOME IN THE TRANSMISSION OF HEREDITARY CHARACTERS.}

By Professor W. J. SpmlmaN, Washington, D. C.

I cannot help thinking that the committee on theoretical research in heredity is one of the most fundamentally important of all those that have been appointed. There is a disposition in some quarters to look upon theoretical research as something impractical and of little value. The fact is, however, that, when our theories have once been brought into accord with fact, they become of the utmost practical importance. If our committee can work out the actual facts concerning the manner in which hereditary characters are transmitted from one generation to the next, it will have rendered a great service to the men who are attempting to produce improved strains of plants and animals.

It seems to me that we ought to call attention in our report to those points requiring further investigation before a complete theory of heredity can be established. For instance, Wilson and a few other investigators have found an extra chromosome in the nuclei of certain animals. This chromosome does not take part in synapsis and therefore does not divide in the first post-synaptic division, but passes into one of the resulting cells. The two cells thus produced therefore have a different organism. They then split into four, two of which have each the extra chromosome. This chromosome is present only in the germ cells produced by the male. It is believed that when the ovum is fertilized by a male cell not having the extra chromosome the resulting chromosome is female; but if fertilized by the germ cell having the extra chromosome the progeny is male. It is easy to see the importance of this point to the practical breeder of animals. If it can be demonstrated that sex is controlled by the presence or absence of a particular chromosome, it settles for all time the question of sex con- 